\title{
De novo HIV infection diagnoses in a Department of Dermatology and Venereology in Lisbon, Portugal
}

\author{
Pedro Mendes-Bastos', Ana Brasileiro', Eugénia Matos-Pires', \\ Isaura Rodrigues², Cristina Marques², Vasco Coelho-Macias' \\ and Cândida Fernandes'
}

\begin{abstract}
Background: Portugal has the highest prevalence rate of HIV infection in Western Europe. The proportion of patients with a late diagnosis, carried out in full-blown AIDS stage, remains high. Skin and mucous membrane manifestations are not rare in these patients.

Objective: A demographic, clinical, and laboratorial characterization of patients with de novo HIV infection diagnosis made in the Department of Dermatology and Venereology of a central hospital in Lisbon, Portugal.

Methods: Retrospective review of medical records of adult patients newly diagnosed with HIV infection (reactive immunoassay for antibodies to HIV-I/HIV-2 or HIV p24 antigen) in the Dermatology and Venereology Department of a Portuguese central hospital in the period between January 2005 and December 2013.

Results: During the study period, 97 new cases were diagnosed, 70 men and 27 women. The median age at diagnosis was 36 years. Of the total, 50 cases were diagnosed with a concomitant sexually transmitted infection (STI), more frequently syphilis and ano-genital HPV infection. The remaining 47 patients were diagnosed with other dermatological conditions such as prurigo nodularis, psoriasis, and Kaposi's sarcoma. The duration of complaints that lead to medical attention ranged from $<1$ week to 8 years, being significantly lower in patients diagnosed with a concomitant STI $(p<0.01)$. Basal viral load was also lower in this group of patients $(p<0.05)$. Of all the new diagnosed cases, $80 \%$ of patients are currently retained in care.

Conclusion: This study grants a descriptive overview of de novo HIV infection diagnoses performed by dermatovenereologists in a central hospital in Lisbon, Portugal. As in the past, the importance of Dermato-Venereology for HIV infection diagnosis remains present in daily clinical practice.
\end{abstract}

\section{Keywords}

AIDS, diagnosis, epidemiology, Europe, HIV

Date received: 22 November 2015; accepted: 13 October 2016

\section{Introduction}

In 1982, the Centers for Disease Control and Prevention put forward the definition of a new disease entity, the acquired immunodeficiency syndrome (AIDS), based on increasing numbers of reports of immunodeficiency-associated disease in previously healthy young men. ${ }^{1}$ In the next years, when it became evident that HIV was primarily sexually transmitted and that more than $95 \%$ of patients had skin manifestations in the course of their disease, ${ }^{2,3}$ dermatologists throughout the world diagnosed and treated many patients with HIV infection or/and AIDS.
Since then, much has changed, and in countries where antiretroviral drugs are widely available, HIV/ AIDS changed from a fatal disease to a chronic disease

\footnotetext{
'Department of Dermatology and Venereology, Centro Hospitalar de Lisboa Central, Lisboa, Portugal

${ }^{2}$ Department of Laboratory Medicine/Clinical Pathology, Centro

Hospitalar de Lisboa Central, Lisboa, Portugal

Corresponding author:

Pedro Mendes-Bastos, Department of Dermatology and Venereology, Alameda de Santo António dos Capuchos - Serviço de Dermatologia, Hospital de Santo António dos Capuchos, Lisboa II50-314, Portugal. Email: pmendesbastos@gmail.com
} 
requiring lifelong medication and medical management in many ways similar to the general population. ${ }^{4,5}$

HIV infection is a major public health concern in Europe, characterized by continuously rising case numbers. The average rate of newly diagnosed HIV infections in Europe was 5.7 cases per 100,000 in 2011. ${ }^{6}$ The average rate of newly diagnosed HIV infections in Portugal is considerably higher, 13.6 cases per 100,000 in 2013. ${ }^{7}$ On the other hand, as far as AIDS is concerned, diagnoses in Europe have been steadily declining (1.2 cases per 100,000 in 2010), but in countries such as Latvia ( 4.8 cases per 100,000), Portugal ( 2.8 cases per $100,000)$, Spain $(1.8$ cases per 100,000), and Estonia (2.8 cases per 100,000$)$, these rates are still hauntingly high. ${ }^{6}$ So, the global impact of new HIV/AIDS diagnoses in Europe is still very worrisome. In order to optimize early HIV detection, the HIV in Europe Initiative has proposed a new approach - the indicator condition-guided HIV testing - encouraging physicians to offer an HIV test to individual patients with certain medical conditions. ${ }^{8-10}$

In a world where prompt HIV diagnosis and treatment are associated with increased longevity and reduced transmission, ${ }^{11,12}$ the paradigm towards this disease has shifted from "damage control" to "the earlier, the better". Besides treating side effects of antiretroviral drug therapies and helping to curb its spread by control of other sexually transmitted infections (STIs), does the dermatovenerologist still contribute to the diagnosis of HIV infection in high resource settings?

The objective of the present study is to elaborate a demographic, clinical, and laboratorial characterization of patients with de novo HIV infection diagnosis made in the Department of Dermatology and Venereology of a central hospital in Portugal.

\section{Materials and methods}

This study is a retrospective review of medical records of patients older than 16 years who were newly diagnosed with HIV infection in General Dermatology Clinic, Urgent Care Dermatology Clinic, Sexually Transmitted Infections Clinic and Dermatology Inpatient Ward of Hospital de Curry Cabral in the period between January 2005 and December 2013 (9 years).

Hospital de Curry Cabral is a central hospital in Lisbon, Portugal, integrated in Centro Hospitalar de Lisboa Central ("Lisbon's Central Hospital Group"), a group of six public cooperating hospitals. General Dermatology Clinic receives patients from local general practitioners and other medical specialties in Centro Hospitalar de Lisboa Central (by appointment only). Urgent Care Dermatology Clinic receives patients from local general practitioners, the emergency department, and other medical specialties for immediate dermatological care. STI Clinic is a walk-in sexual health clinic and no referral from other physician is needed. Dermatology inpatient ward receives admitted patients from all Dermatology Clinics and emergency department. In Portugal, as in most European countries, Dermato-Venereology is a medical-surgical specialty that provides both dermatological and STI care. Thus, not only are dermatovenereologists responsible for the Dermatology Clinics but also for the STI Clinic and both areas of care are practised in an integrated way.

Laboratorial screening was made with reactive chemiluminescent microparticle immunoassay for antibodies to HIV-1, HIV-2, or HIV p24 antigen ARCHITECT HIV Ag/Ab Combo, Abbot ${ }^{\circledR}$. According to institution protocol, all positive screening results are confirmed by HIV-1 and HIV-2 Western Blot on a second serum specimen. The term "de novo HIV infection" refers to newly diagnosed HIV infections in patients that were until that moment unaware of having this condition.

Statistical analysis was performed using Chi-squared test, Fisher's exact test and Student's $t$ test. Significance was defined for $p<0.05$.

\section{Results}

During the period from January 2005 to December 2013 (nine years), the serology for HIV1/2 was requested 3833 times by dermatovenereologists in the Dermatology and Venereology Department. A total of 97 individuals tested positive for HIV (3\%), of which 93 were HIV-1 and 4 HIV-2 infections. The yearly average of new HIV diagnoses in this department was 11 cases per year. When considering the newly diagnosed HIV patients $(n=97), 45$ attended the STI Clinic $(46 \%), 27$ the GD Clinic $(28 \%), 21$ the UCD Clinic (22\%), and 4 were admitted to the DIW (4\%).

There were 70 male $(72 \%)$ and 27 female patients $(28 \%)$. As for the place of birth, 49 patients $(51 \%)$ were born in Portugal and the remaining 48 patients were migrants, of which 38 from African countries.

About half of the patients $(n=50 / 97)$ were diagnosed with a concomitant STI (Figure 1). In this group, the top five conditions were recent or secondary syphilis (18 cases), ano-genital human papilloma virus (HPV) infection (13 cases), Neisseria gonorrhoeae infection (5 cases), ano-genital herpes simplex virus (HSV) infection (4 cases), and Molluscum contagiosum infection (4 cases). Figure 2 includes all STI diagnosis made in this group.

For patients diagnosed with a non-STI dermatological condition ( $n=47 / 97$, Figure 1$)$, the top five diagnoses were prurigo nodularis (seven cases), psoriasis 


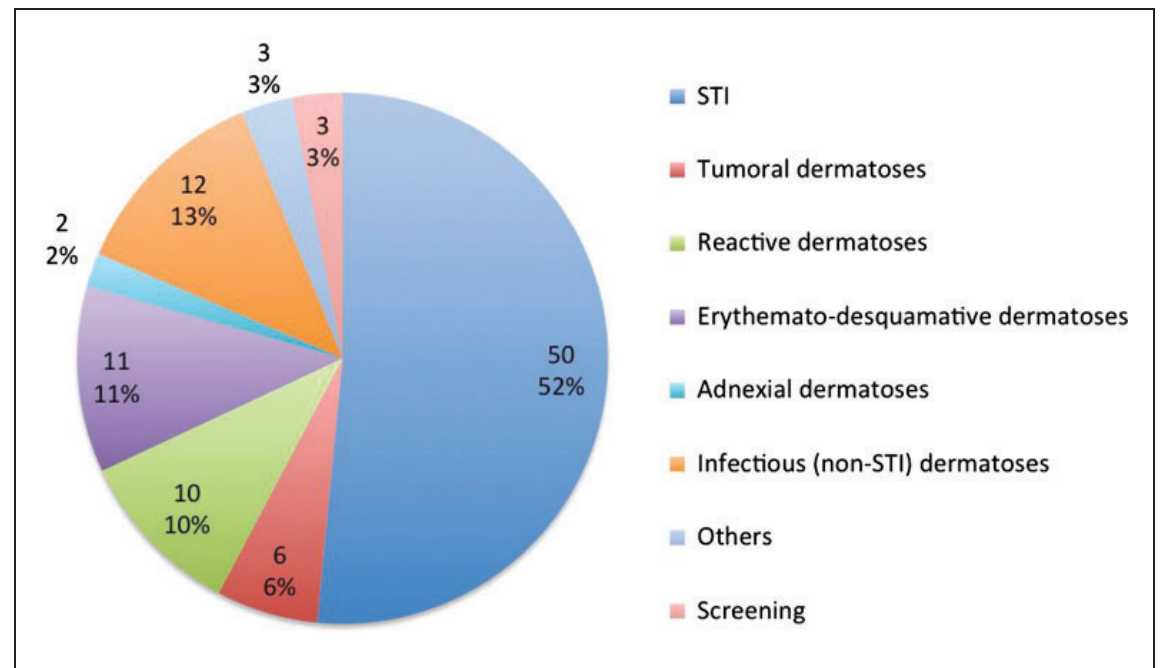

Figure I. Distribution of concomitant dermatovenereological diagnoses in patients with de novo HIV infection identified in the Dermatology and Venereology Department of Hospital de Curry Cabral, Lisbon (Portugal) between 2005 and 2013 ( $n=97$ ).

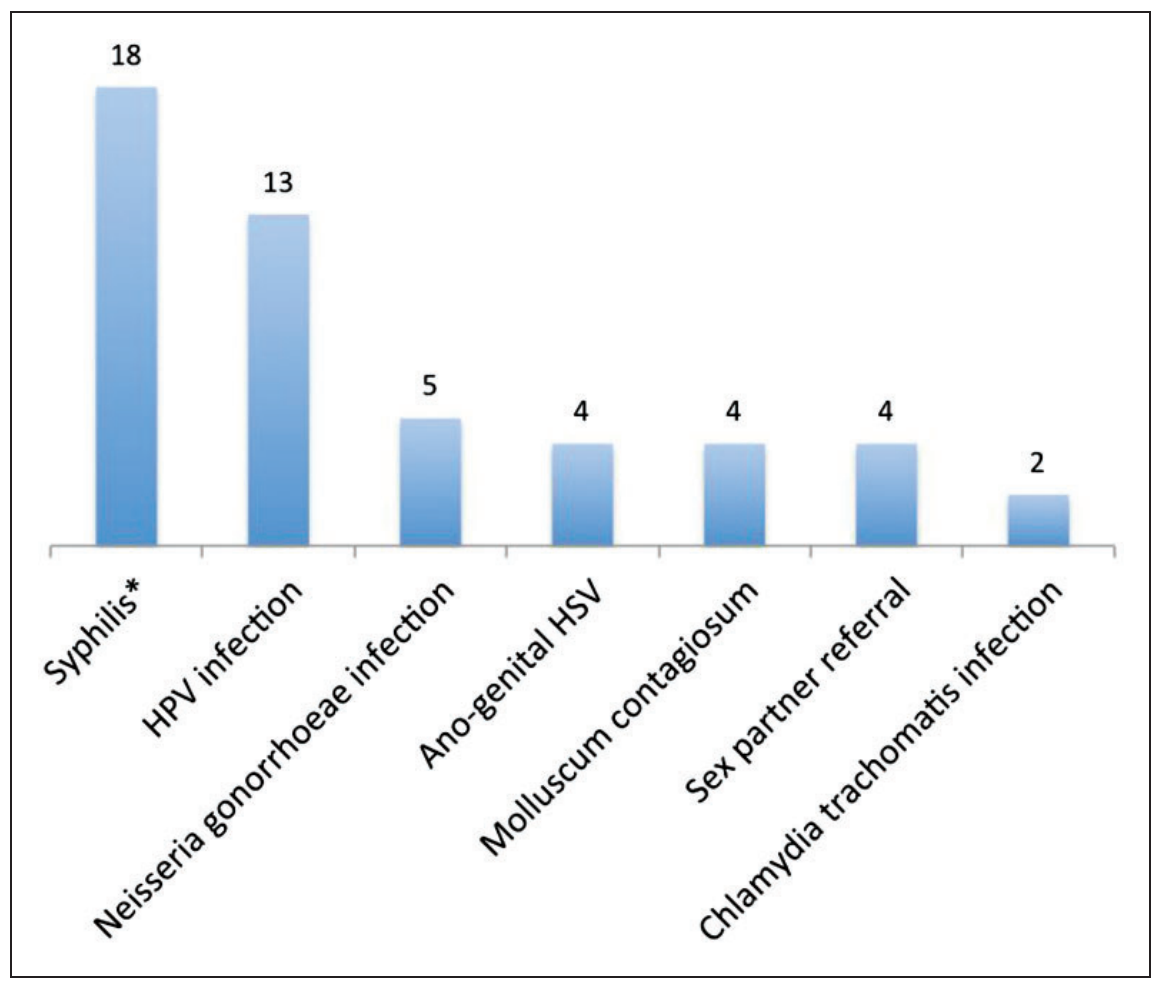

Figure 2. Distribution of concomitant STI diagnosed in patients with de novo HIV infection $(n=50)$. *Nine cases of secondary syphilis and nine of recent syphilis.

(five cases), Kaposi's sarcoma (four cases), varicellazoster virus (VZV) infection (four cases), and oral HSV infection (three cases). The full list is extensive and is summarized in Figure 3.

The duration of complaints that lead to dermatovenereological attention ranged from $<1$ week to 8 years (median 12 weeks). For patients diagnosed with a concomitant STI, the median duration of complaints was 4 weeks, whereas it was 16 weeks for those with other dermatological diagnoses $(p<0.01)$.

Most diagnosed patients ( $85 \%$ ) fall in the age group between 21 and 50 years old (range 17 to 73 years old), 


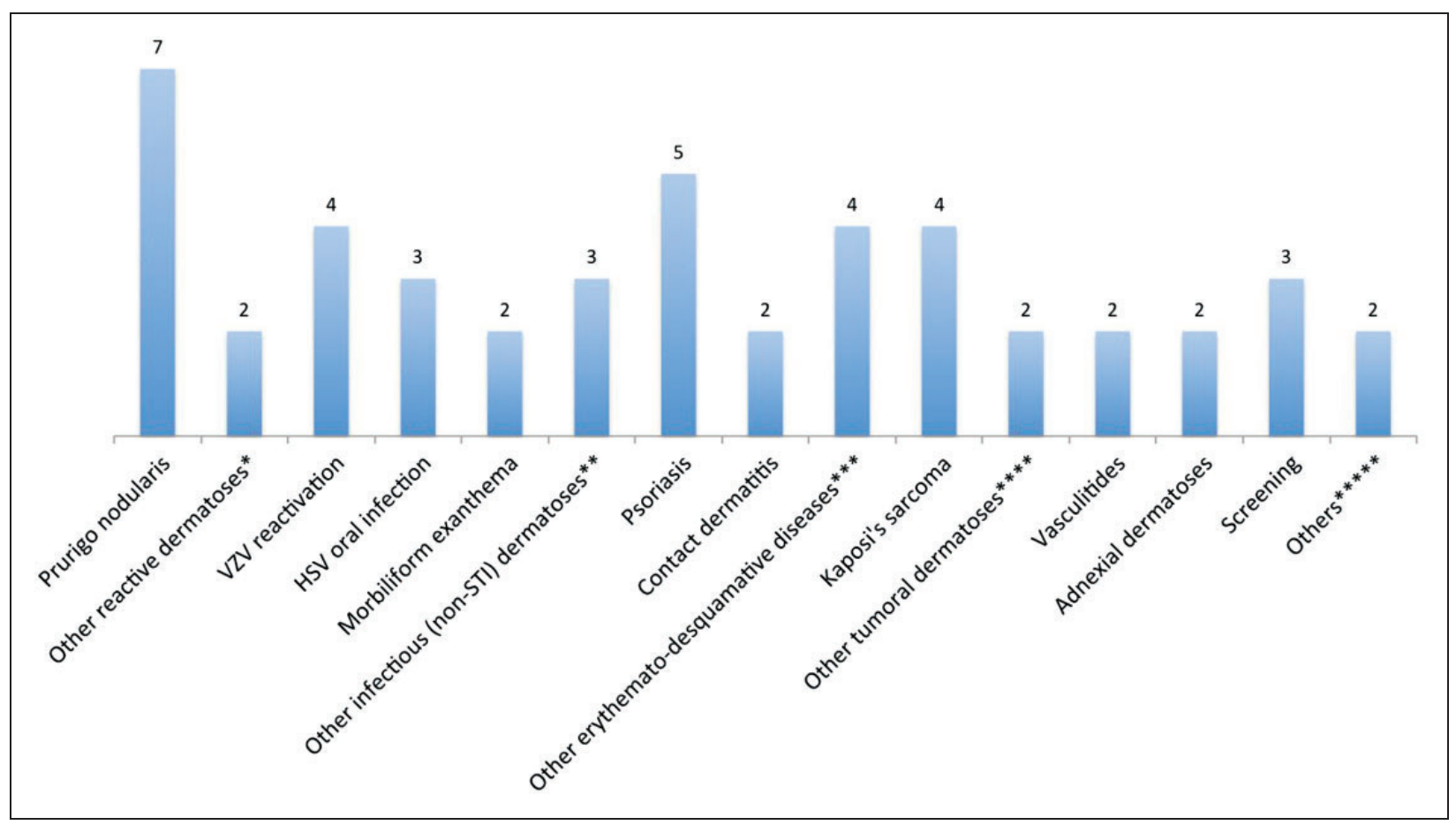

Figure 3. Distribution of non-STI dermatoses identified in patients with de novo HIV infection $(n=47)$.

*Granuloma annulare, eosinophilic folliculitis (one case each).

**Tinea corporis, impetigo, recurrent erysipelas (one case each).

***Seborrhoeic dermatitis, generalized dermatitis, lichen sclerosus, lichen simplex cronicus (one case each).

****B-cell cutaneous lymphoma, oral florid papillomatosis (one case each).

******Perniosis, porphyria cutanea tarda.

with a median age at diagnosis of 36 years old. Patients diagnosed with a STI were younger than those diagnosed with other conditions (median age 36 and 46 years old, respectively, $p<0.01$ ).

Overall, $33(34 \%)$ patients had an initial CD4 cell count lower than 200 cells $/ \mathrm{mm}^{3}, 24(25 \%)$ had between $200-500$ cells $/ \mathrm{mm}^{3}$ and $17(18 \%)$ had over 500 cells/ $\mathrm{mm}^{3}$ (data not available for 23 patients). There was no significant difference in CD4 cell count of patients diagnosed with a concomitant STI when compared to those having a non-STI condition $(p=0.259)$.

Data on basal viral load were available for 71 patients, of which 23 had over 1000 copies $/ \mathrm{mL}$, while 48 patients had less than that value. Of note, patients diagnosed with an STI had lower viral load count than those having a non-STI diagnosis $(p<0.01)$.

As far as follow-up is concerned, $12 \%$ of all de novo HIV diagnosed patients (12/97 cases) never returned after the initial GD/UCD/STI appointment (even after telephone summoning). These patients were not informed of the diagnosis by the dermatovenereologist, nor were they referred to Infectious Diseases (ID) Clinic. All the patients that did return to the department ( $88 \%$, i.e. $85 / 97$ cases) were informed of the diagnosis by their dermatovenereologist and were referred to ID Clinic; 8\% (7/97 cases) never attended that Clinic. The total "lost-to-care" patients were 19: 11 patients from STI Clinic and 8 patients from Dermatology Clinics. Overall, the retention in care rate is of $80 \%$ (78/97 cases).

\section{Discussion}

Tschachler has recently reminded us of the importance of Dermato-Venereology in the clinical management of HIV patients. ${ }^{3}$ Concrete data regarding the role of dermatovenereologists in diagnosing new HIV infection cases today in Europe are unavailable; current data from Portugal concerning this matter will be the object of this discussion.

A total of 97 de novo HIV infection diagnoses were made, which corresponds to an overall $3 \%$ positivity rate regarding the 3833 times dermatovenereologists requested HIV testing. Opposed to what might seem expected at first glance, most new HIV diagnoses in this Department were made in the Dermatology Clinics/DIW (54\%) and not in the STI Clinic (46\%). However, one must take into account that STI were also diagnosed at Dermatology Clinics, making concomitant STI the most representative diagnostic 
group $(52 \%)$. When comparing these two groups regarding the duration of complaints that lead to dermatovenereological attention, the median duration of the clinical picture was significantly shorter for STI diagnoses (4 weeks) than for the remaining patients (16 weeks) $(p<0.01)$. Analyzing the concomitant conditions in each group can help justify this difference in the duration of complaints: the second group of patients were diagnosed mostly cutaneous manifestations of advanced immunosuppression, while the STI group had just concomitant STIs. These data confirm the importance of testing for HIV all attendees presenting with an STI. From our experience, exuberant, difficult to treat or atypical presentations of diseases like prurigo nodularis, herpetic infections or psoriasis should also lead to promptly exclude an underlying HIV infection. The re-emergence of syphilis and frequent coinfection with HIV must also be highlighted. It is not surprising to see that patients diagnosed with non-STI conditions had higher median number of HIV-1 RNA copies/mL $(p<0.05)$ when compared to patients diagnosed in STI Clinics; in fact, the median CD4 T cell count tended to be lower in the non-STI conditions group and most of these cases were AIDS diagnoses.

Comparing the top conditions observed in our Department with the indicator conditions in the HIV in Europe Initiative Guidance, ${ }^{8}$ several common diagnoses can be found (including Kaposi's sarcoma, herpetic infections, severe or recalcitrant psoriasis or STI). Champenois et al. ${ }^{13}$ also show that under current screening policies, missed opportunities for HIV testing remain unacceptably high in France, recognizing that HIV testing should be offered to patients presenting with HIV-related cutaneous conditions and/or STI is pointed as crucial to make earlier HIV diagnoses. In our opinion, both the public and the medical community in general are not as aware as they should be regarding HIV-related cutaneous diseases. This point may explain the longer duration of complaints and more advanced immunosuppression found in the patients diagnosed in the Dermatology Clinics/DIW as a non-dermatovenereologist doctor had previously observed and referred all patients.

Prurigo nodularis was the most frequent diagnosis in our series. Recent studies by Iking et al. ${ }^{14}$ (Germany) and Tan and Tey ${ }^{15}$ (Singapore) evaluated series of prurigo nodularis patients regarding coexisting diseases and no case of HIV infection was found. ${ }^{14,15}$ Magand et al. ${ }^{16}$ (French Guyana), however, showed that prurigo nodularis was predictive of advanced immunosuppression in their case series. Prurigo nodularis was not included in the HIV in Europe Initiative Guidance Guidelines and maybe these new data will draw the whole medical community's attention to this dermatological condition.
Most of the newly diagnosed HIV individuals were male, both in STI and non-STI groups $(70 \%$ in each group). It is known that the major attendees of STI Clinic are men, and that asymptomatic STIs are more frequent in women and one could easily speculate about other possible motifs to justify these numbers. Whatever reasons for this difference might there be, it is interesting to notice this fact and perhaps have a lower index of suspicion for males, should this information be confirmed in other studies in dermatology clinics.

The high number of new diagnoses in migrant patients $(50 \%$ of all cases) must be highlighted. In fact, considering the global numbers of new cases in Europe, the figure in some populations such as those from sub-Saharan African countries is a group of special concern due to median lower CD4 $\mathrm{T}$ cell counts at the time of diagnosis and higher percentage of AIDS cases. ${ }^{6}$ The population served by our Department is mainly urban (center of Lisbon) and culturally and ethnically diverse, including many immigrants from the Portuguese-speaking African countries. Whether the HIV infection was acquired in Africa or in Europe is a matter of controversy and beyond the point of discussion in this paper; instead, we wonder if this more advanced state of immunosuppression due to HIV in this population could be justified by social fragility and difficult access to health care. In a fast-changing Europe, socially and culturally enriched by intense migration flows, the relevance of these findings could not be more contemporary.

Of all the new HIV patients diagnosed by dermatovenereologists in the mentioned period, the retention in care rate is of $80 \%$ (78/97 cases), similar to other recent European publications. ${ }^{17}$

In our experience, dermatovenereologists still play a pivotal role in the clinical management of HIV-infected patients, from the earliest phase of diagnosis to the treatment period, and also in the promotion of healthy sexual practices. In a dynamic and changing world, struggling against the HIV/AIDS epidemic, the importance of Dermato-Venereology holds an undeniable position.

\section{Acknowledgments}

The authors gratefully acknowledge the help of Beatriz Nunes, António P Matos, Simão Rodeia, João B Furtado, and Marta Jonet in the gathering of the data.

\section{Declaration of conflicting interests}

The authors declared no potential conflicts of interest with respect to the research, authorship, and/or publication of this article.

\section{Funding}

The authors received no financial support for the research, authorship, and/or publication of this article. 


\section{References}

1. Current trends update on acquired immune deficiency syndrome (AIDS) - United States. MMWR 1982; 31: 277-279.

2. Tschachler E, Bergstresser PR and Stingl G. HIV-related skin diseases. Lancet 1996; 348: 659-663.

3. Tschachler E. The dermatologist and the HIV/AIDS pandemic. Clin Dermatol 2014; 32: 286-289.

4. Bogner J. HIV infection: chronic disease with comorbidities. Internist 2012; 53: 1169-1178.

5. Buckhold F 3rd. Primary care of the human immunodeficiency virus patient. Med Clin North Am 2015; 99: 1105-1122.

6. European Centre for Disease Prevention and Control (ECDC). Annual epidemiological report. Reporting on 2011 surveillance data and 2012 epidemic intelligence data, www.ecdc.europa.eu (2013, accessed 26 October 2016).

7. DGS (Direção Geral da Saúde). Portugal - infeção VIH, SIDA e tuberculose em números 2014. Programa Nacional para a Infeção VIH/SIDA, www.dgs.pt (accessed 26 October 2016).

8. HIV in Europe Initiative. HIV indicator conditions: guidance for Implementing HIV testing in adults in health care settings, 2012.

9. Raben D, Mocroft A, Rayment M, et al. Auditing HIV testing rates across Europe: results from the HIDES 2 study. PLoS One 2015; 10: e0140845.
10. Sullivan A, Raben D, Reekie J, et al. Feasibility and effectiveness of indicator condition-guided testing for HIV: results from HIDES I (HIV indicator diseases across Europe study). PLoS One 2013; 8: e52845.

11. Marks G, Crepaz N and Janssen RS. Estimating sexual transmission of HIV from persons aware and unaware that they are infected with the virus in the USA. AIDS 2006; 20: 1447-1450.

12. Nakagawa F, Lodwick RK, Smith CJ, et al. Projected life expectancy of people with HIV according to timing of diagnosis. AIDS 2012; 26: 335-343.

13. Champenois K, Cousien A, Cuzin L, et al. Missed opportunities for HIV testing in newly-HIV-diagnosed patients, a cross sectional study. BMC Infect Dis 2013; 13: 200.

14. Iking A, Grundmann S, Chatzigeorgakidis E, et al. Prurigo as a symptom of atopic and non-atopic diseases: aetiological survey in a consecutive cohort of 108 patients. J Eur Acad Dermatol Venereol 2013; 27: 550-557.

15. Tan W and Tey H. Extensive prurigo nodularis: characterization and etiology. Dermatology 2014; 228: 276-280.

16. Magand F, Nacher M, Cazorla C, et al. Predictive values of prurigo nodularis and herpes zoster for HIV infection and immunosuppression requiring HAART in French Guiana. Trans R Soc Trop Med Hyg 2011; 105: 401-404.

17. Van Beckhoven D, Lacor P, Moutschen M, et al. Factors associated with the continnuum of care of HIV-infected patients in Belgium. J Int AIDS Soc 2014; 17(Suppl 3): 19534. 